\title{
더ㄴㅓㅐ Genesis
}

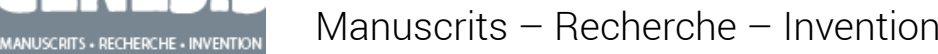

$44 \mid 2017$

Après le texte

\section{L'activité éditoriale dans le processus créatif de textes littéraires}

Alberto Cadioli

\section{(2) OpenEdition}

1 Journals

Édition électronique

URL : https://journals.openedition.org/genesis/1734

DOI : 10.4000/genesis. 1734

ISSN : 2268-1590

Éditeur :

Presses universitaires de Paris Sorbonne (PUPS), Société internationale de génétique artistique littéraire et scientifique (SIGALES)

Édition imprimée

Date de publication : 9 mai 2017

Pagination : $57-64$

ISBN : 979-1023-105636

ISSN : $1167-5101$

\section{Référence électronique}

Alberto Cadioli, «L'activité éditoriale dans le processus créatif de textes littéraires », Genesis [En ligne], 44 | 2017, mis en ligne le 15 mai 2018, consulté le 18 mars 2023. URL : http://

journals.openedition.org/genesis/1734; DOI : https://doi.org/10.4000/genesis.1734

Tous droits réservés 


\title{
L'activité éditoriale dans le processus créatif de textes littéraires*
}

\author{
Alberto Cadioli
}

L 'attention particulière portée aux implications du travail éditorial dans le processus de lecture des textes littéraires ${ }^{1}$ a été étendue, ces dernières années, aux interventions de l'instance éditoriale dans le processus créatif lui-même. On doit cette extension à de nouvelles réflexions dans le domaine de la critique génétique ${ }^{2}$, en France, et de la philologie, en Italie (en particulier de la philologie qu'on appelle «d'auteur $\left.{ }^{3} »\right)$.

Dans la présentation du numéro 41 de Genesis «Auteur(s) et acteurs de la genèse» - Nicolas Donin et Daniel Ferrer soulignent que «l'écrivain qui rature un mot et le remplace n'est pas exactement le même que celui qui l'a inscrit, même dans le cas d'une correction intervenant presque instantanément. Si celui qui relit avait exactement la même perspective que celui qui a écrit, il n'y aurait pas de ratures, pas de corrections, pas de nouvelles versions.
C'est l'intervalle qui s'ouvre entre les deux positions qui crée l'espace dans lequel peut opérer la critique génétique ${ }^{4}$ ». Et ils ajoutent, en citant Valéry, que l'œuvre «est le fruit d'une collaboration d'états très divers ». On pourrait en dire autant des interventions des éditeurs sur l'écriture des auteurs.

Si la plupart des recherches historiques concernant ce rapport se réfèrent aux premiers siècles de l'impression 5 , nous porterons notre attention dans cet article sur le XIX et surtout le $\mathrm{XX}^{\mathrm{e}}$ siècle. Durant cette période, les auteurs comme les acteurs du travail éditorial manifestèrent une nouvelle conscience de leur rôle respectif.

Siegfried Unseld - l'un des éditeurs littéraires les plus importants de l'Allemagne du XXe siècle, en tant que directeur de la maison d'édition Suhrkamp Verlag - affirme explicitement dans la première édition de son ouvrage

(*) Ce travail a été réalisé avec le soutien du laboratoire d'excellence TransferS (programme Investissements d'avenir ANR-10-IDEX-0001-02PSL* et ANR-10-LABX-0099).

1. Depuis longtemps, les implications du travail éditorial dans la lecture et dans l'interprétation des textes littéraires sont proposées par les études d'histoire du livre, de bibliographie matérielle, de sociologie de la communication. On ne peut rappeler ici que quelques titres tirés de ces différents domaines : Donald F. McKenzie, Bibliography and sociology of texts, London, British Library, coll. «The Panizzi Lectures », 1986 (Cambridge, Cambridge University Press, 1999); Roger Chartier, Culture écrite et société. L'ordre des livres (XIVe et XVIIIe siècle), Paris, Albin Michel, coll. «Bibliothèque histoire», 1996; Roger Chartier, La Main de l'auteur et l'esprit de l'imprimeur, XVIe-XVIIIe siècle, Paris, Gallimard, coll. «Folio histoire», 2015 ; Brigitte Ouvry-Vial, Anne Réach-Ngô (dir.), L’Acte éditorial. Publier à la Renaissance et aujourd'hui, Paris, Classiques Garnier, coll. «Pratiques éditoriales », 2010.

2. Le volume n ${ }^{\circ} 41$ de Genesis (2015) - «Créer à plusieurs mains », dir. Nicolas Donin et Daniel Ferrer - propose un long essai d’Anne Réach-Ngô, «Du texte au livre, et retour : la production littéraire à la Renaissance, une création collaborative?» (p. 29-47), qui introduit une réflexion sur la «création collaborative» et sur la «participation de l'instance éditoriale à la création littéraire» : "Qualifier d' "écriture" l'intervention des hommes du livre sur le texte qu'ils éditent - tant par sa mise en forme typographique et spatiale que par l'ajout de péritextes - revient [...] à reconnaître leur pleine participation à la littérarité de l'œuvre» (ibid., p. 32). Voir aussi Anne Réach-Ngô (dir.), «Genèses éditoriales », dossier thématique de la revue Seizième Siècle, $\mathrm{n}^{\circ} 10,2014$.

3. Nous nous permettons de renvoyer, à propos des études italiennes, à Alberto Cadioli, Le diverse pagine. Il testo letterario fra scrittore, editore, lettore, Milano, il Saggiatore, coll. «La cultura », 2012. Voir aussi Paola Italia, Editing Novecento, Roma, Salerno, coll. «Strumenti per l'università », 2013 et Paola Italia, Giorgio Pinotti (dir.), Editori e filologi. Per una filologia editoriale, coll. «Studi (e testi) italiani», Roma, Bulzoni, 2014.

4. Nicolas Donin et Daniel Ferrer, «Auteur(s) et acteurs de la genèse», dans Genesis, n 41, op. cit., p. 24. Sur ce sujet on peut voir aussi Daniel Ferrer, Logiques du brouillon. Modèles pour une critique génétique, Paris, Les Éditions du Seuil, coll. «Poétique», 2011.

5. Voir à ce propos Anne Réach-Ngô, L'Écriture éditoriale à la Renaissance. Genèse et promotion du récit sentimental français (1530-1560), Genève, Droz, coll. «Travaux d'Humanisme et Renaissance», 2013, et Anne Réach-Ngô (dir.), « Genèses éditoriale», op. cit. 
L'Auteur et son éditeur (dont le chapitre significatif était «La mission de l'éditeur de textes littéraires»), que l'éditeur est «le premier partenaire» de l'auteur6. Unseld précise qu'il pense à ces éditeurs «qui sont le reflet de l'histoire littéraire ou culturelle de leur temps, ou qui déterminent ses orientations ${ }^{7}$ ». On pourrait ajouter que la «mission» de l'éditeur est celle d'intervenir dans la culture et dans la littérature de son temps, endossant un rôle qu'on qualifierait de «militant». Italo Calvino a défini lui-même le consultant éditorial comme étant celui «qui travaille avec l'objectif que la culture de son temps ait une image spécifique plutôt qu' une autre 8 ». Par rapport à son objectif, il croise nécessairement l'écriture de ceux qui soumettent leurs textes à la lecture éditoriale de la maison d'édition Einaudi, où Calvino a été rédacteur et lecteur éditorial de 1947 jusqu'à sa mort, en 1985.

Les points de vue d'Unseld et de Calvino ouvrent à une meilleure compréhension du travail des éditeurs face à l'écriture des auteurs, et permettent de mieux cerner les changements que les éditeurs ont introduits, ce qu'ils ont suggéré, modifié, réécrit, en intervenant dans les processus créatifs de textes des XIXe et XXe siècles.

Le mot «éditeur» renvoie en réalité à différentes entités éditoriales : le directeur de la maison d'édition, les lecteurs qui expriment un premier jugement sur les textes proposés à la publication, le directeur d'une collection, les rédacteurs ou les consultants qui ont été chargés d'éditer les textes; c'est-à-dire des personnes précises, bien identifiables, qui possèdent des convictions littéraires, stylistiques, linguistiques spécifiques.

On retrouve fréquemment, dans les archives des maisons d'édition, les traces des processus éditoriaux modernes, parce que la plupart des éditeurs sont intervenus sur le texte : leurs suggestions, voire les modifications qu'ils apportent, apparaissent pendant l'écriture du texte sous la forme de requêtes plus ou moins directives apparaissant sur le manuscrit définitif, ou une fois l'écriture achevée, lors de la préparation typographique et de la correction des épreuves.

Les cas qui pourraient être rappelés sont nombreux, mais certains exemples - tous tirés de la littérature italienne - sont très remarquables, car ils permettent d'identifier des situations paradigmatiques et de leur donner une étiquette spécifique, dans un jeu taxinomique teinté d'ironie.

\section{La créativité face à l'éditeur entrepreneur}

En 1873, l'éditeur florentin Gasparo Barbèra demande par lettre à Edmondo De Amicis (écrivain déjà bien connu grâce à ses contes et à ses reportages) de développer le texte décrivant la Hollande qu'il est en train d'achever.

Après avoir précisé l'importance de la longueur du texte par rapport aux lecteurs, l'éditeur propose d'insérer des pages aussi bien sur la Belgique ( $\ll$ Et avec la Hollande, est-ce que vous pourriez dire quelque chose à propos de la Belgique $^{9}$ ?») que sur les paysages hivernaux («un connaisseur me disait que sans avoir vu la Hollande en hiver on ne peut pas parler de la Hollande $10_{»}$ ). L'auteur accepte toutes les suggestions et continue à écrire selon les conseils de son éditeur. L'intervention de Barbèra a des mobiles commerciaux, mais De Amicis n'a aucune réticence à les prendre en compte : il représente en effet ces nouveaux auteurs de la seconde moitié du XIXe siècle, qui désirent désormais être lus par le plus grand nombre.

Il est vrai que le texte imprimé par Barbèra est le résultat de l'intervention de l'éditeur, mais peut-on toutefois parler de deux auteurs, d'une création «à deux mains»? Cette question pourrait se poser pour tous les exemples qui suivent.

6. Siegfried Unseld, Der Autor und sein Verleger, Suhrkamp Verlag, Frankfurt am Main, 1978, éd. fr. : L'Auteur et son éditeur, Paris, Gallimard, coll. «Le monde actuel», 1983, p. 40. Le titre de ce premier écrit, en italien, est : Les Devoirs de l'éditeur littéraire. On sait bien que le mot «mission» a des connotations très différentes par rapport à celles du mot «devoir».

7. Ibid., p. 17.

8. «uno che lavora [...] a far sì che la cultura del suo tempo abbia un volto piuttosto che un altro»; lettre de Italo Calvino à Carlos Alvarez, datée du 5 octobre 1964, dans Italo Calvino, I libri degli altri. Lettere 1947-1981, a cura di Giovanni Tesio, con una nota di Carlo Fruttero, Torino, Einaudi, coll. «I coralli», 1991, p. 465.

9. «E coll'Olanda non può dare qualche cenno sul Belgio ?»; lettre de Gasparo Barbèra à Edmondo De Amicis, datée du 19 août 1873, dans Lettere di Gaspero Barbèra. Tipografo editore (1841-1879), pubblicate dai figli con prefazione di Alessandro d'Ancona, Firenze, Barbèra, 1914, p. 290.

10. «un intelligente mi diceva che senza veder l'Olanda d'inverno non si può parlar dell'Olanda» (ibid.). 


\section{La créativité face à l'éditeur «partenaire»}

Giovanni Verga ne parvenait pas à décider quelle préface publier des deux qu'il avait écrites pour son roman I Malavoglia : il les envoie, toutes deux, à son éditeur, Emilio Treves; celui-ci exprime sa préférence, mais il transpose des phrases de l'une à l'autre préface et en crée donc une troisième, finalement publiée. L'auteur accepte le nouveau texte, qui n'est pas le sien, mais qui le devient puisqu'il en assume la paternité face au lecteur, par l'édition de son roman.

Les corrections qui ont été suggérées à Eugenio Montale par le responsable, chez Mondadori, en 1977, de l'édition du recueil Quaderno di quattro anni (édition française : Cahier de quatre ans), appartiennent au domaine de la poésie. Grâce à elles, d'autres observations peuvent être présentées à propos de différentes volontés de l'écrivain. Domenico Porzio (l'un des dirigeants de la maison d'édition et ami du poète) suggère, pendant la correction des épreuves du recueil, de modifier certains vers ${ }^{11}$. Il note ses suggestions, au crayon, dans les marges blanches des épreuves, et, dans un deuxième temps, il ajoute, à l'encre bleue ou verte, les réponses du poète, qui sont presque toutes négatives, sauf dans quelques cas intéressants. Par exemple, dans le poème Leggendo Kasafis (En lisant Kasafis) - dont le premier titre, «Casafis», a été corrigé sur les épreuves et sera de nouveau corrigé par le poète dans l'édition critique en 1980 - l'expression du vers 17 dans le tapuscrit et dans les premières et deuxièmes épreuves, «tagliarsi le vene [se couper les veines]», a été changée, selon la proposition de l'éditeur, en «tagliarmi le vene [me couper les veines]». La correction modifie sans aucun doute le sens, mais l'écrivain est d'accord. Toutefois, en relisant ses textes en vue de l'édition critique, quelques années plus tard, il en revient à sa première formulation 12 .

Dans le même recueil, l'éditeur pose une question concernant la graphie du mot «scoiattoli» (écureuils), qui en italien peut être écrit avec un $i$ ou un $j$. Une annotation dans les premières épreuves du poème Una città del Nord (Une ville $d u$ Nord) témoigne que le poète laisse le choix à son éditeur. Les deuxièmes épreuves portent encore un $j$, mais dans les troisièmes un correcteur anonyme de la maison d'édition modifie, à l'encre rouge, le $j$ en $i$; la nouvelle graphie se place «à jamais » dans le livre imprimé.

Les épreuves du Cahier deviennent donc le journal de la relation entre le poète et son éditeur dans la phase précédant l'impression et donnent un témoignage très intéressant sur cette étape de l'écriture, gardant la trace des corrections et contre-corrections apportées par le poète.

L'éditeur de poésie Vanni Scheiwiller offre, à son tour, un remarquable exemple de collaboration créatrice.

En 1954, Scheiwiller lit dans une revue littéraire le poème de Clemente Rebora, qui n'écrivait et ne publiait presque plus rien depuis qu'il avait embrassé la vie religieuse. L'éditeur, bien qu'encore très jeune, adresse une lettre à l'illustre poète par laquelle il l'invite à écrire une Via Crucis en vers. Rebora accueille favorablement la suggestion et interrompt son long silence poétique grâce à cette sollicitation : la publication de Via Crucis (1955) inaugure une nouvelle période créative consacrée à la poésie religieuse, brève (le poète mourra en 1957) mais très riche.

La collaboration de Scheiwiller est aussi à l'origine, en 1963, du recueil Nel magma (Dans le magma), qui témoigne d'un important changement dans la poétique de Mario Luzi, un des plus importants poètes italiens du $\mathrm{XX}^{\mathrm{e}}$ siècle. Dans ce cas également, c'est l'éditeur qui, suite à la lecture de quelques vers du poète dans une revue littéraire, propose à celui-ci la publication d'un bref recueil. Luzi, qui avait le projet d'insérer ces poèmes dans un ouvrage différent dont il avait déjà tracé un sommaire, fait sienne l'idée de l'éditeur, écrit d'autres vers et publie finalement un petit livre à l'identité forte.

\section{Hommes de lettres éditeurs}

Durant le $\mathrm{XX}^{\mathrm{e}}$ siècle, nombreux sont les écrivains qui ont travaillé au sein de maisons d'édition en Europe et aux États-Unis (rappelons seulement le nom de Thomas Stearns Eliot, directeur chez Faber and Faber) et qui, en tant qu' «éditeur», ont modifié les textes d'écrivains à partir d'une idée personnelle de la littérature et du style.

11. L'histoire de ces corrections a été présentée dans Alberto Cadioli, Le diverse pagine, op. cit., p. 101-102, à partir des épreuves consultées dans les archives de la Fondazione Arnoldo e Alberto Mondadori, à Milan (Fondo Domenico Porzio).

12. Eugenio Montale, L'opera in versi, a c. di Gianfranco Contini e Rosanna Bettarini, Torino, Einaudi, 1980, coll. «I millenni», p. 1117. 
En Italie, le cas d'Elio Vittorini est très significatif, celuici étant à la fois homme de lettres, écrivain et directeur de la collection de romans «I gettoni» chez Einaudi; à la même période (les années cinquante), il était également consultant de la maison d'édition Mondadori. Vittorini était très déterminé en corrigeant les textes publiés dans sa collection; et il était créatif, intervenant directement ou suggérant des changements selon son opinion littéraire personnelle et son expérience d'écriture ${ }^{13}$.

Après avoir lu les pages narratives de Beppe Fenoglio, Vittorini refusa le roman La paga del sabato (éd. fr. : La Paie du samedi), mais accepta les contes, qui auraient dû être publiés sous le titre I ventitré giorni della città di Alba (éd. fr. : Les Vingt-Trois Jours de la ville d'Albe). Il suggéra cependant de nombreuses modifications, qui avaient pour objectif de mettre en relief une littérature capable de s'opposer à la représentation de la réalité, qu'il jugeait sans caractère, de nombreux écrivains après la Seconde Guerre mondiale.

Une lettre de Fenoglio révèle que furent introduits dans la structure du livre de nouveaux épisodes, qui étaient des chapitres de La Paie du samedi transformés en brefs contes, selon la demande de Vittorini. Les lettres de l'auteur montrent qu'il avait même effacé tout ce que l'éditeur avait condamné comme «écriture banale 14 ».

Le recueil Les Vingt-Trois Jours de la ville d'Albe fut donc publié en 1952 après les modifications que l'auteur même avait introduites dans la structure et dans le style, mais qui avaient été proposées par le directeur de la collection. Il est intéressant de noter à ce stade que Calvino, en tant que rédacteur de la maison d'édition Einaudi, avait lu, lui aussi, le roman et les contes de Fenoglio, et qu'il ne partageait pas du tout les opinions de Vittorini. Il n'aurait pas refusé le roman, qu'il préférait aux contes, il n'aurait pas suggéré de rendre autonomes des chapitres en les transformant en contes, il n'aurait pas demandé de modifier le style dans le sens «barbare» que Vittorini avait indiqué à l'auteur.

Le travail de Calvino auprès de la maison d'édition Einaudi permet d'ajouter d'autres exemples concernant les interventions personnelles des éditeurs qui, en tant qu'hommes de lettres engagés dans la culture de leur temps, contribuent à forger la langue littéraire d'une période en participant à l'écriture d'autres écrivains.
Calvino, en particulier, en envoyant des «lettres d'éditeur» aussi bien à des écrivains dont les romans avaient été refusés qu'à ceux qui étaient en train de publier leurs textes, donnait des conseils et suggérait des changements qui venaient de ses convictions littéraires. Voici un exemple représentatif de son activité d'homme de lettres éditeur : en écrivant à un auteur, dont le roman avait été accepté par la maison d'édition, Calvino suggère d'effacer «tout ce qui a un ton "poétique" et "profond" et qui au contraire est terriblement banal 15 ». Il ajoute qu'il faut proposer, dans les récits, des événements, et refuser absolument tout lyrisme. C'est une idée fondamentale et récurrente aussi bien dans les pages de poétique de Calvino que dans toutes ses interventions éditoriales.

Une lettre que Calvino adresse à Leonardo Sciascia en 1962, pendant la publication du roman Il consiglio d'Egitto (éd. fr. : Le Conseil d'Égypte), suggère à l'auteur des changements affectant les métaphores : «il faut que le niveau des métaphores ait une cohérence et une harmonie; sinon l'écriture est fortuite, journalistique ${ }^{16_{»}}$.

L'idée de littérature qui guide le travail de Calvino écrivain - la recherche d'un style personnel et d'une cohérence stylistique - dirige également son travail éditorial. Jugeant «mauvais» le premier chapitre du roman de Pier Paolo Pasolini Una vita violenta (Une vie violente), il affirme (dans une lettre adressée à un rédacteur de la maison d'édition Garzanti, qui venait d'éditer le roman) qu'il aurait fallu l'effacer «sans aucune perte 17 ». Si Une vie violente eût été publié chez Einaudi, on n'aurait jamais lu les pages du

13. Voir à ce sujet Gian Carlo Ferretti, L'editore Vittorini, Torino, Einaudi, coll. «Piccola biblioteca Einaudi », 1992.

14. Les lettres qui concernent la publication des Vingt-Trois Jours de la ville d'Albe, datées de 1950 à 1952, sont rassemblées dans Vito Camerano, Raffaele Crovi, Giuseppe Grasso (dir.), La storia dei «Gettoni » di Elio Vittorini, con la collaborazione di Augusta Tosone, introduzione e note di Giuseppe Lupo, Milano, Aragno, coll. «Biblioteca Aragno », 2007, vol. I, p. 351-374.

15. «tutto quello che c'è di "poetico" e "profondo" e che invece è terribilmente di second'ordine »; lettre d'Italo Calvino à Raffaello Brignetti, datée du 15 janvier 1959, dans Italo Calvino, I libri degli altri, op. cit., p. 285.

16. «il piano delle metafore deve avere una sua coerenza, una sua armonia, se no è scrittura casuale, giornalistica»; lettre d'Italo Calvino à Leonardo Sciascia, datée du 5 octobre 1962, ibid., p. 410.

17. Voir la lettre d'Italo Calvino à Pietro Citati, datée du 8 juin 1959 , ibid., p. 314. 
premier chapitre, que Calvino aurait éliminées ou demandé à l'auteur d'éliminer. Devrait-on parler du «hasard éditorial» dans l'histoire des auteurs et de leurs textes?

L'auteur de Il giardino dei Finzi-Contini (éd. fr. : Le Jardin des Finzi-Contini), George Bassani, a été directeur de la collection de romans italiens contemporains chez Feltrinelli; alors qu'il était en train de corriger, d'un point de vue linguistique, Il gattopardo (éd. fr. : Le Gattopardo) de Giuseppe Tomasi di Lampedusa, en 1958, il ne cachait pas tout ce que ce travail de correction avait d'épuisant : «il faut prêter attention à toutes les broutilles, c'est presque comme pour écrire 18 ». La réécriture éditoriale des auteurs-éditeurs est un sujet qui mériterait approfondissement.

\section{Les interventions des lecteurs de maisons d'édition}

Il est d'ailleurs important de pouvoir identifier les corrections suggérées par les lecteurs de la maison d'édition ${ }^{19}$. Giuseppe Pontiggia, qui était un écrivain raffiné, raconte qu'il écrivait souvent à l'auteur du texte qu'il venait d'expertiser en tant que consultant de maisons d'édition, en lui indiquant les parties les plus faibles, «toujours selon mon goût 20 ».

Lorsque Vincenzo Consolo, surtout connu pour son roman Il sorriso dell'ignoto marinaio (éd. fr. : Le Sourire du marin inconnu), propose à la maison d'édition Mondadori son premier ouvrage, La ferita dell'aprile (La Blessure d'avril), le lecteur en charge de la lecture du texte (l'écrivain Raffaele Crovi) lui demande une révision attentive. Face au «désordre logique» (pour reprendre la formule de Crovi) la révision réclamée devait porter sur les occurrences des expressions dialectales, des expressions argotiques, des dictons siciliens, pour donner au texte une linéarité de discours qui, selon le lecteur de la maison d'édition, aurait amélioré l'œuvre dans un contexte «descriptif-représentatif 21 ». La lecture éditoriale suggérait même l'élimination de deux dernières pages du tapuscrit qui diluaient le sens du roman, «compte rendu d" "un voyage pour sortir de l'enfance/adolescence" 22 ».

Consolo pouvait-il accepter les conseils de son éditeur dès lors qu'ils relevaient d'une idée de récit linéaire, qui n'était pas la sienne ? Cet exemple exprime parfaitement la situation la plus fréquente dans une maison d'édition, qui revient à proposer à l'auteur des changements après l'avoir au préalable assuré de sa volonté de le publier.
Dans quelle mesure les écrivains auxquels leurs éditeurs ont suggéré des modifications au fur et à mesure du processus d'écriture ont-ils changé leurs textes? La critique génétique et la philologie d'auteur ont le devoir de repérer des documents qui pourront le dire. Pour mieux comprendre l'entrecroisement entre écriture d'auteur et demandes de corrections d'éditeur, il faudrait recourir à la collation des pages dactylographiées, des épreuves, du texte imprimé, lorsqu' on a la possibilité de le faire, en tenant compte de toute la documentation existante.

\section{Demandes éditoriales et réécriture des auteurs}

Pendant la publication du roman Ragazzi di vita (éd. fr. : Les Ragazzi), de Pier Paolo Pasolini, le propriétaire de la maison d'édition, Livio Garzanti, craignant la censure de l'Italie des années cinquante, demande à l'auteur de supprimer les nombreux mots vulgaires. Pasolini travaille trois jours sans interruption, mais, en effaçant les gros mots, corrige son écriture et présente finalement un roman profondément modifié, qui offre le même récit, mais dans un registre de langue et un style très différents ${ }^{23}$, résultat d'une nouvelle phase de création et conséquence des «pressions »

18. «bisogna stare attenti alle minuzie quasi come per scrivere»; lettre de Giorgio Bassani à sa femme, datée du 7 juillet 1958, dans Corriere della sera, 12 avril 2001

19. À propos de la lecture éditoriale, voir Ouvry-Vial, «Le savoir lire de l'éditeur? Présupposés et Modalités », dans Bertrand Legendre et Christian Robin (dir.), Figures de l'éditeur. Représentations, savoirs, compétences, territoires, Paris, Nouveau Monde Éditions, 2005.

20. «sempre facendo riferimento al mio gusto»; Giuseppe Pontiggia, «Ragioni e ragionevolezza delle mode letterarie», entretien dans Panta, $\mathrm{n}^{\circ} 19,2001$, p. 102.

21. Raffaele Crovi, «Vincenzo Consolo La ferita dell'aprile» [lecture éditoriale], dans Il mestiere di leggere. La narrativa italiana nei pareri di lettura della Mondadori (1950-1971), a c. di Annalisa Gimmi, Milano, Fondazione Arnoldo e Alberto Mondadori/il Saggiatore, 2002, p. 268. 22. "resoconto di "un viaggio d'uscita dall'infanzia/adolescenza”", ibid., p. 269.

23. La première rédaction, malheureusement, n'a jamais été publiée, mais elle est présentée par les pages qui analysent Ragazzi di vita dans l'édition scientifique des œuvres complètes de Pasolini. Voir Pier Paolo Pasolini, Romanzi e racconti, a c. di Walter Siti e Silvia De Laude, con due saggi di Walter Siti, cronologia a cura di Nico Naldini, Milano, Mondadori, coll. «I Meridiani », 1998, vol. I. 
de l'éditeur. Il n'est pas question d'évaluer la qualité littéraire des deux rédactions mais de souligner qu'elles portent, l'une et l'autre, le même titre, bien qu'on puisse considérer qu'il ne s'agisse plus du même roman, les changements ayant impliqué le texte dans sa totalité.

\section{Interventions éditoriales «forcées»}

Les exemples développés jusqu'à présent proposent différentes typologies d'interventions éditoriales dans le processus créatif, mais, dans tous les cas évoqués, les auteurs ont accepté les observations et les modifications des éditeurs, et souvent ont ouvert de nouveau le chantier de l'écriture.

Parfois, en revanche, l'intervention éditoriale peut être «forcée». Un témoignage très significatif d'un tel mode d'intervention, même si l'écrivain a assisté au processus de révision, est offert par le récit-pamphlet Eros e Priapo. Da furore a cenere (éd. fr. : Éros et Priape. De la fureur aux cendres), de Carlo Emilio Gadda, un des plus importants écrivains italiens du $\mathrm{XX}^{\mathrm{e}}$ siècle. Son écriture a connu une longue histoire, avant la publication en 196724 . La première rédaction date de 1944-1946, mais elle était demeurée inédite, car les éditeurs craignaient les conséquences de la violence politique du récit (qui était centré sur le régime fasciste et la figure de Mussolini) et de l'obscénité dans les descriptions des fascistes et de leur guide politique. Pendant les années cinquante, Gadda en publia quelques extraits dans une revue littéraire, avant de décider d'abandonner un texte sur lequel il était revenu plusieurs fois. Pressé par Livio Garzanti de donner un livre à sa maison d'édition, car l'écrivain n'avait pas respecté son contrat en publiant La cognizione del dolore (La Connaissance de la douleur) chez Einaudi, Gadda lui envoie Éros et Priape, dans une rédaction qu'il avait récemment corrigée; après lecture, l'éditeur demande à Enzo Siciliano (un jeune écrivain ayant déjà publié des contes) d'intervenir pour en éliminer la violence et l'obscénité.

Gadda accepte tous les choix pris au fur et à mesure de la révision, en restant indifférent aux modifications; il en résulte un texte très différent par rapport à celui que Gadda avait écrit et réécrit pendant vingt ans, un texte qui a perdu sa violence originelle, principalement dans la langue. On pourrait dire que l'auteur a participé à la création d'une œuvre qui n'était pas la sienne; c'est pour cette raison qu'on a parlé d' «édition "quasi" posthume» et d' «édition d'auteur forcée 25 ». La première rédaction, qui a été retrouvée récemment, révèle en effet que l'on doit désormais parler de deux livres : l'un qui a été publié sous le nom de l'auteur, mais qui a été réécrit par un éditeur; l'autre qui est demeuré inédit, bien qu'il s'agisse de la rédaction originale.

Un second exemple soulignera combien peut être utile l'examen des interventions «forcées» et directement introduites par l'éditeur, même si l'auteur les a acceptées. Pour expliquer le retard dans la publication de Il maestro di Vigevano (éd. fr. : L'Instituteur de Vigevano) de Lucio Mastronardi, Calvino, qui était en train d'en suivre l'édition auprès de la maison d'édition Einaudi, écrivait explicitement : «Nous avions accepté de publier ce livre parce qu'il contient des pages talentueuses, pleines de force poétique et d'humour, mais nous avions demandé à Lucio la permission de supprimer des pages qui n'étaient pas publiables. Lucio était d'accord pour que nous fassions nous-mêmes ce travail [...], mais une attentive révision a retardé la publication ${ }^{26}$.»

\section{L'auteur et son texte}

La réécriture au sein des maisons d'édition pose de nouveau la question de l'idée de littérature, d'écriture, de récit que l'homme de lettres éditeur, le lecteur de la maison d'édition, le directeur de celle-ci ou le directeur d'une collection veulent présenter aux lecteurs, et des relations entre la création de l'auteur et l'activité de l'éditeur.

Il est vrai qu'après avoir pris connaissance de l'avis de l'éditeur, les auteurs peuvent «l'accepter, et modifier leur

24. L'histoire de la genèse, de l'écriture du texte, de la publication de Éros et Priape. De la fureur aux cendres est développée par Paola Italia et Giorgio Pinotti, «Edizioni d'autore coatte : il caso di "Eros e Priapo" (con l'originario primo capitolo, 1944-46)», Ecdotica, n 5 , 2008, p. 7-102. 25. Ibid., p. 137 , n. 38.

26. «Avevamo accettato questo libro per la pubblicazione, perché contiene pagine piene di talento e di forza poetica e di humour, ma avevamo chiesto a Lucio di poter tagliare altre pagine che non erano pubblicabili. Lucio aveva acconsentito che noi facessimo questo lavoro, [...] ma una attenta revisione ha ritardato naturalmente la pubblicazione »; lettre d'Italo Calvino à Maria Mastronardi, datée du 24 novembre 1961, dans I libri degli altri, op. cit., p. 386. 
œuvre dans la direction suggérée par ce jugement, ou se rebeller contre lui, quand le rapport de force, institutionnel ou affectif, leur permet de le faire. Dans les deux cas, cette confrontation axiologique (c'est-à-dire ayant trait aux valeurs et à leurs hiérarchies) aura joué un rôle important 27 ».

Naturellement la question prioritaire, quand l'auteur permet toutes les corrections, voire la réécriture, volontairement ou à contrecœur, concerne l'auctorialité. Qui est l'auteur des textes imprimés qui ont été évoqués ci-dessus? Peut-on réellement parler de création «à plusieurs mains 28 »?

Nous voici parvenus au stade des réflexions théoriques, qui tournent autour du problème de l'auteur, de son identité, voire de son existence.

Le mythe romantique de l'auteur solitaire et qui écrit sous la seule inspiration n'intéresse pas la philologie d'auteur italienne, à laquelle ses pages appartiennent; elle n'a pas non plus de proximité avec les débats des années soixante et soixante-dix concernant la mort ou la survivance de l'auteur, la possibilité ou non de repérer l'intention de celui qui a écrit le texte (l'intentio auctoris), la transformation de l'auteur dans une figure abstraite produite par le texte même ou par son édition. La philologie d'auteur fait porter son attention sur la connaissance du processus déclenché par l'acte d'écrire, individuel dans la plupart des cas, et dont le résultat est un texte ayant presque toujours été altéré pendant sa première édition ou par les éditions publiées à travers les décennies (ou à travers les siècles).

La question n'est donc pas : qui est l'auteur? mais : peut-on connaître un texte dans l'état où celui qui a écrit a voulu, ou aurait voulu, le donner aux lecteurs?

Dès lors qu'un texte a eu un état initial, sur lequel de nombreux acteurs ont encore travaillé, on peut chercher à identifier ce qui a été écrit en premier, puis reconnaître les différentes mains qui sont intervenues sur le texte au cours de sa première publication ou des publications successives. On pourrait utiliser à ce propos une image de Daniel Ferrer concernant l'écriture : il faut «gratter les fresques pour retrouver la sinople ${ }^{29}$ », et affirmer la nécessité, revendiquée par la critique génétique et par la philologie d'auteur (bien que pour des mobiles différents : l'histoire de l'écriture, d'un côté, et l'histoire d'un texte pour son édition critique, de l'autre), d'identifier les instances multiples qui révèlent «la sédimentation des volontés diverses » et «temporellement disjointes ${ }^{30} »$, aussi bien celles de l'écrivain que de l'éditeur.
Face aux textes contemporains, donc, l'étude des pages qui portent la trace des corrections ou de la réécriture des éditeurs est très importante, afin de savoir qui a écrit quoi, de repérer les choix propres à la maison d'édition, voire une poétique éditoriale, ou d'approfondir par des témoignages peu connus les opinions littéraires d'hommes de lettres qui ont travaillé comme éditeurs.

À ce stade, d'autres questions doivent être posées. Siegfried Unseld, à propos de l'éditeur, ajoutait : c'est le premier «qui juge le manuscrit, qui apprécie l'éventuel travail à effectuer sur ce manuscrit pour l'amener à atteindre en substance et en qualité ce degré maximal qu' est capable de lui donner un auteur ${ }^{31}$ ». Quel est l'itinéraire emprunté par l'éditeur afin qu'un texte arrive à son « degré maximal», et comment peut-il l'identifier? À partir de quel point de repère peut-on parler de «degré maximal» auquel l'écrivain aurait dû ou aurait pu arriver?

Évidemment, on ne peut donner de réponses valables pour toutes les situations textuelles et éditoriales, surtout parce que chaque réponse implique une notion de «qualité » qu'il est impossible de fixer dans l'abstrait. Toutefois, l'identification de la main de l'écrivain et de la main de l'éditeur (ou de la main de l'auteur et de l'esprit de l'imprimeur, pour citer Roger Chartier ${ }^{32}$ ) peut indiquer des horizons différents, et, pour ce qui concerne le domaine de la philologie d'auteur, poser une dernière question qui est la première d'un nouveau territoire d'investigation : comment éditer les textes modifiés par les éditeurs 33 ?

27. Nicolas Donin et Daniel Ferrer, «Auteur(s) et acteurs de la genèse», art. cité, p. 15.

28. Pour toutes les questions et tous les problèmes qui concernent ce sujet, il est nécessaire d'avoir comme point de repère le numéro 41 (2015) de Genesis, «Créer à plusieurs mains », particulièrement l'article déjà cité de Nicolas Donin et Daniel Ferrer.

29. Daniel Ferrer, «Le Pays des trente-six mille volontés, ou "tu l'auras voulu"», dans Ecdotica, n 8, 2011, p. 99.

30. Nicolas Donin et Daniel Ferrer, «Auteur(s) et acteurs de la genèse », art. cité, p. 24.

31. Siegfried Unseld, L'Auteur et son éditeur, op. cit., p. 40.

32. Roger Chartier, La Main de l'auteur et l'esprit de l'imprimeur, op. cit. 33. La contribution de Bénédicte Vauthier se confronte à cette question, voir p. 39. 
Alberto CAdioli est professeur de littérature italienne contemporaine à l'Université de Milan. Dans ses derniers travaux, il s'occupe de philologie et de transmission de textes littéraires. Parmi ses publications, on peut citer : Le diverse pagine. Il testo letterario tra autore, editore, lettore (Milano, il Saggiatore, 2012) et Letterati editori. Attività editoriale e modelli letterari nel novecento (Milano, il Saggiatore, 2017).

alberto.cadioli@unimi.it

\section{Résumés \\ L'activité éditoriale dans le processus créatif de textes littéraires}

La demande de corrections qu'un éditeur soumet à un écrivain avant l'impression de son texte et plus encore les interventions qu'introduisent directement les rédacteurs sur le texte en cours d'édition agissent sur le processus de création littéraire et sur l'identité d'une œuvre. Le présent article propose une typologie des demandes des éditeurs et des réponses des écrivains, que les changements soient accueillis comme d'utiles conseils, ou qu'ils soient acceptés sans qu'un consensus évident se soit manifesté, ou même contre leur gré. Les nombreux exemples proposés montrent que la volonté de l'auteur et celle de l'éditeur s'opposent souvent, de sorte que la publication d'une œuvre peut être le résultat d'un affrontement entre des poétiques divergentes : celle que l'auteur a en vue et celle que l'éditeur propose (et diffuse à travers ses publications).

The requests for corrections that a publisher makes to a writer before publishing his/her text, and even more, the interventions that editors insert in the text during its preparation, affect the process of literary creation and the work's identity. This article suggests a typology of publishers' requests and writers' answers, whether the changes be received as useful advice or accepted without evident consensus or even against the writers' wishes. The many examples presented show that the author's will and that of the publisher are often opposed, so that the publication of a work can be the result of a confrontation between two divergent poetics: the one the author has in mind and the one the publisher suggests (and releases with its publications).

Wenn der Herausgeber eines Textes von einem Autor vor der Drucklegung Korrekturen verlangt, und mehr noch bei Eingriffen eines Redakteurs in einen Text im Zuge der Edition, wird der literarische Schaffensprozess und die Identität eines Werkes beeinflusst. Der vorliegende Artikel präsentiert eine Typologie von Änderungsvorschlägen von Seiten der Editoren und Antworten von Autoren: sei es, dass die Änderungen als nützliche Ratschläge aufgenommen werden, sei es, dass sie ohne explizite Zustimmung akzeptiert werden, sei es gar, dass kein Einverständnis des Autors erfolgt. Unsere zahlreichen Beispiele zeigen, dass es oft einen Gegensatz zwischen dem Willen des Autors und jenem des Herausgebers gibt, sodass die Publikation eines Werkes das Resultat des Aufeinandertreffens divergierender poetischer Bestrebungen sein kann: die Poetik des Autors und die Poetik, die der Verlag vorschlägt (und in seinen Publikationen verbreitet)
Las correcciones que un editor solicita a un escritor antes de imprimir su texto y, más aún, las intervenciones directas de los redactores en el texto que se está editando, influyen en los procesos de la creación literaria y en la identidad de una obra. El presente artículo propone una tipología de los pedidos de los editores y de las respuestas de los escritores, tanto cuando los cambios han sido considerados por éstos como consejos útiles, como cuando han sido aceptados sin que un consenso evidente se haya puesto de manifiesto o contra su voluntad. Los numerosos ejemplos propuestos muestran que los designios del escritor se oponen a menudo a los del editor, de modo que la publicación de una obra puede ser el resultado de la confrontación de dos poéticas divergentes: la que el autor prevé y la que el editor propone (y difunde a través de sus publicaciones).

A aplicação de correções que um editor solicita ao escritor antes de imprimir o seu texto, bem como as intervenções directas dos editores sobre o texto em curso de edição, têm peso no processo da criação literária e na identidade da obra. Este artigo propõe uma tipologia dos pedidos dos editores e das respostas dos escritores, que umas vezes são bem acolhidos, outras não. Muitos exemplos mostram que a vontade do autor e a do editor muitas vezes se opõem, de modo que a publicação da obra pode ser resultado do confronto de duas poéticas divergentes: o que o autor tem em vista e o que o editor oferece (e difunde nas suas publicações).

La richiesta di correzioni che un editore sottopone a uno scrittore prima della stampa del suo testo, e ancora di più gli interventi direttamente portati dai redattori su un testo in lavorazione in casa editrice agiscono sul processo di creazione letteraria e sull'identità di un'opera. Lo scritto qui presentato offre una tipologia delle richieste degli editori e delle risposte degli scrittori, sia quando i cambiamenti vengono accolti come utili consigli, sia quando vengono accettati senza la manifestazione di un evidente consenso o addirittura forzatamente. I numerosi esempi proposti testimoniano come spesso volontà dell'autore e volontà dell'editore si oppongano, così che la pubblicazione di un'opera può essere il risultato del confronto tra poetiche diverse: quella perseguita dall'autore e quella proposta (e diffusa attraverso le sue pubblicazioni) dall'editore. 